\title{
A new version of the Gleason-Kahane-Żelazko theorem in complete random normed algebras
}

Yuehan Tang ${ }^{1,2}$

Correspondence: tangjohn@126. com

${ }^{1}$ LMIB and School of Mathematics and Systems Science, Beihang University, Beijing 100191, P. R. China

Full list of author information is available at the end of the article

\section{Abstract}

In this article we first present the notion of multiplicative $L^{0}$-linear function.

Moreover, we establish a new version of the Gleason-Kahane-Żelazko theorem in unital complete random normed algebras.

Mathematics Subject Classification 2000: 46H25; 46H05; $15 \mathrm{~A} 78$.

Keywords: random normed module, random normed algebra, multiplicative $L^{0}$-linear function, Gleason-Kahane-Żelazko theorem.

\section{Introduction}

Gleason [1] and, independently, Kahane and Żelazko [2] proved the so-called GleasonKahane-Żelazko theorem which is a famous theorem in classical Banach algebras. There are various extensions and generalizations of this theorem [3]. The GleasonKahane-Żelazko theorem in an unital complete random normed algebra as a random generalization of the classical Gleason-Kahane-Żelazko theorem is given in [4].

Based on the study of [5], we will establish a new version of the Gleason-KahaneŻelazko theorem in an unital complete random normed algebra. In this article we first present the notion of multiplicative $L^{0}$-functions. Then, we give the new version of the Gleason-Kahane-Żelazko theorem in an unital complete random normed algebra as another random generalization of the classical Gleason-Kahane-Żelazko theorem.

The remainder of this article is organized as follows: in Section 2 we give some necessary definitions and lemmas and in Section 3 we give the main results and proofs.

\section{Preliminary}

Throughout this article, $N$ denotes the set of positive integers, $K$ the scalar field $R$ of real numbers or $C$ of complex numbers, $\bar{R}$ (or $[-\infty,+\infty]$ ) the set of extended real numbers, $(\Omega, \mathcal{F}, P)$ a probability space, $\overline{\mathcal{L}}^{0}(\mathcal{F}, R)$ the set of extended real-valued $\mathscr{F}$-random variables on $\Omega, \bar{L}^{0}(\mathcal{F}, R)$ the set of equivalence classes of extended real-valued $\mathscr{F}$-random variables on $\Omega, \mathcal{L}^{0}(\mathcal{F}, K)$ the algebra of $K$-valued $\mathscr{F}$-random variables on $\Omega$ under the ordinary pointwise addition, multiplication and scalar multiplication operations, $L^{0}(\mathcal{F}, K)$ the algebra of equivalence classes of $K$-valued $\mathscr{F}$-random variables on $\Omega$, i.e., the quotient algebra of $\mathcal{L}^{0}(\mathcal{F}, K)$, and 0 and 1 the null and unit elements, respectively.

(c) 2012 Tang; licensee Springer. This is an Open Access article distributed under the terms of the Creative Commons Attribution License (http://creativecommons.org/licenses/by/2.0), which permits unrestricted use, distribution, and reproduction in any medium, provided the original work is properly cited. 
It is well known from [6] that $\bar{L}^{0}(\mathcal{F}, R)$ is a complete lattice under the ordering $\leq: \xi$ $\leq \eta$ iff $\xi^{0}(\omega) \leq \eta^{0}(\omega)$ for $P$-almost all $\omega$ in $\Omega$ (briefly, a.s.), where $\xi^{0}$ and $\eta^{0}$ are arbitrarily chosen representatives of $\xi$ and $\eta$, respectively. Furthermore, every subset $A$ of $\bar{L}^{0}(\mathcal{F}, R)$ has a supremum, denoted by $\vee A$, and an infimum, denoted by $\wedge A$, and there exist two sequences $\left\{a_{n}, n \in N\right\}$ and $\left\{b_{n}, n \in N\right\}$ in $A$ such that $\mathrm{V}_{n \geq 1} a_{n}=\mathrm{V} A$ and $\Lambda_{n \geq 1}$ $b_{n}=\Lambda A$. If, in addition, $A$ is directed (accordingly, dually directed), then the above $\left\{a_{n}\right.$, $n \in N\}$ (accordingly, $\left\{b_{n}, n \in N\right\}$ ) can be chosen as nondecreasing (accordingly, nonincreasing). Finally $L^{0}(\mathcal{F}, R)$, as a sublattice of $\bar{L}^{0}(\mathcal{F}, R)$, is complete in the sense that every subset with an upper bound has a supremum (equivalently, every subset with a lower bound has an infimum).

Specially, let $\bar{L}_{+}^{0}(\mathcal{F})=\left\{\xi \in \bar{L}^{0}(\mathcal{F}, R) \mid \xi \geq 0\right\}$ and $L_{+}^{0}(\mathcal{F})=\left\{\xi \in L^{0}(\mathcal{F}, R) \mid \xi \geq 0\right\}$.

The following notions of generalized inverse, absolute value, complex conjugate and sign of an element in $L^{0}(\mathcal{F}, K)$ bring much convenience to this article.

Definition 2.1. [7] Let $\xi$ be an element in $L^{0}(\mathcal{F}, K)$. For an arbitrarily chosen representative $\xi^{0}$ of $\xi$, define two $\mathscr{F}$-random variables $\left(\xi^{0}\right)^{-1}$ and $\left|\xi^{0}\right|$, respectively, by

$$
\left(\xi^{0}\right)^{-1}(\omega)= \begin{cases}\frac{1}{\xi^{0}(\omega)} & \text { if } \xi^{0}(\omega) \neq 0 \\ 0, & \text { otherwise }\end{cases}
$$

and

$$
\left|\xi^{0}\right|(\omega)=\left|\xi^{0}(\omega)\right|, \quad \forall \omega \in \Omega .
$$

Then the equivalence class of $\left(\xi^{0}\right)^{-1}$, denoted by $\xi^{-1}$, is called the generalized inverse of $\xi$; the equivalence class of $\left|\xi^{0}\right|$, denoted by $|\xi|$, is called the absolute value of $\xi$. When $\xi \in L^{0}(\mathcal{F}, C)$, set $\xi=u+i v$, where $u, v \in L^{0}(\mathcal{F}, R), \bar{\xi}:=u-i v$ is called the complex conjugate of $\xi$ and $\operatorname{sgn}(\xi):=|\xi|^{-1} \cdot \xi$ is called the sign of $\xi$. It is obvious that $|\xi|=|\bar{\xi}|, \xi \cdot \operatorname{sgn}(\bar{\xi})=|\xi|,|\operatorname{sgn}(\xi)|=\tilde{I}_{A}, \xi^{-1} \cdot \xi=\xi \cdot \xi^{-1}=\tilde{I}_{A}$, where $A=\left\{\omega \in \Omega: \xi^{0}\right.$ $(\omega) \neq 0\}$ and $\tilde{I}_{A}$ denotes the equivalence class of the characteristic function $I_{A}$ of $A$. Throughout this article, the symbol $\tilde{I}_{A}$ is always understood as above unless stated otherwise.

Besides the equivalence classes of $\mathscr{F}$-random variables, we also use the equivalence classes of $\mathscr{F}$-measurable sets. Let $A \in \mathcal{F}$, then the equivalence class of $A$, denoted by $\tilde{A}$, is defined by $\tilde{A}=\{B \in \mathcal{F}: P(A \Delta B)=0\}$, where $A \Delta B=(A \backslash B) \cup(B \backslash A)$ is the symmetric difference of $A$ and $B$, and $P(\tilde{A})$ is defined to be $P(A)$. For two $\mathscr{F}$-measurable sets $G$ and $D, G \subset D$ a.s. means $P(G \backslash D)=0$, in which case we also say $\tilde{G} \subset \tilde{D} ; \tilde{G} \cap \tilde{D}$ denotes the the equivalence class determined by $G \cap D$. Other similar notations are easily understood in an analogous manner.

As usual, we also make the following convention: for any $\xi, \eta \in L^{0}(\mathcal{F}, R), \xi>\eta$ means $\xi \geq \eta$ and $\xi \neq \eta ;[\xi>\eta]$ stands for the equivalence class of the $\mathscr{F}$-measurable set $\left\{\omega \in \Omega: \xi^{0}(\omega)>\eta^{0}(\omega)\right\}$ (briefly, $\left[\xi^{0}>\eta^{0}\right]$ ), where $\xi^{0}$ and $\eta^{0}$ are arbitrarily selected representatives of $\xi$ and $\eta$, respectively, and $I_{[\xi>\eta]}$ stands for $\tilde{I}_{\left[\xi^{0}>\eta^{0}\right]}$. If $A \in \mathcal{F}$, then $\xi$ $>\eta$ on $\tilde{A}$ means $\xi^{0}(\omega)>\eta^{0}(\omega)$ a.s. on $A$, similarly $\xi \neq \eta$ on $\tilde{A}$ means that $\xi^{0}(\omega) \neq \eta^{0}(\omega)$ a.s. on $A$, also denoted by $\tilde{A} \subset[\xi \neq \eta]$. 
Definition 2.2. [7] An ordered pair $(S,\|\cdot\|)$ is called a random normed module (briefly, an RN module) over $K$ with base $(\Omega, \mathcal{F}, P)$ if $S$ is a left module over the algebra $L^{0}(\mathcal{F}, K)$ and $\|\cdot\|$ is a mapping from $S$ to $L_{+}^{0}(\mathcal{F})$ such that the following conditions are satisfied:

$(\mathrm{RNM}-1)\|\xi x\|=|\xi|\|x\|, \forall \xi \in L^{0}(\mathcal{F}, K), x \in S$

(RNM-2) $\|x+y\| \leq\|x\|+\|y\|, \forall x, y \in S$;

(RNM-3) $\|x\|=0$ implies $x=0$ (the zero element in $S$ ).

Where $\|x\|$ is called the $L^{0}$-norm of the vector $x$ in $S$.

In this article, given an RN module $(S,\|\cdot\|)$ over $K$ with base $(\Omega, \mathcal{F}, P)$ it is always assumed that $(S,\|\cdot\|)$ is endowed with its $(\epsilon, \lambda)$-topology: for any $\epsilon>0,0<\lambda<1$, let $N(\epsilon, \lambda)=\{x \in S \mid P\{\omega \in \Omega:\|x\|(\omega)<\epsilon\}>1-\lambda\}$, then the family $\mathcal{U}_{0}=\{N(\varepsilon, \lambda) \mid \varepsilon>0,0<\lambda<1\}$ forms a local base at the null element 0 of some metrizable linear topology for $S$, called the $(\epsilon, \lambda)$-topology for $S$. It is well known that a sequence $\left\{x_{n}, n \geq 1\right\}$ in $S$ converges in the $(\epsilon, \lambda)$-topology to some $x$ in $S$ if $\left\{\left\|x_{n}-x\right\|\right.$, $n \geq 1$ \} converges in probability $P$ to 0 , and that $S$ is a topological module over the topological algebra $L^{0}(\mathcal{F}, K)$, namely the module multiplication $\cdot: L^{0}(\mathcal{F}, K) \times S \rightarrow S$ is jointly continuous (see [7] for details). Besides, let $L^{0}(\mathcal{F}, K)$ be the RN module of equivalence classes of $X$-valued $\mathscr{F}$-random variables on $(\Omega, \mathcal{F}, P)$, where $X$ is an ordinary normed space, then it is easy to see that the $(\epsilon, \lambda)$-topology on $L^{0}(\mathcal{F}, K)$ is exactly the topology of convergence in probability and $L^{0}(\mathcal{F}, K)$ is complete iff $X$ is complete, in particular $L^{0}(\mathcal{F}, K)$ is complete.

Definition 2.3. [5] An ordered pair $(S,\|\cdot\|)$ is called a random normed algebra (briefly, an RN algebra) over $K$ with base $(\Omega, \mathcal{F}, P)$ if $(S,\|\cdot\|)$ is an RN module over $K$ with base $(\Omega, \mathcal{F}, P)$ and also a ring such that the following two conditions are satisfied:

(1) $(\xi \cdot x) y=x(\xi \cdot y)=\xi \cdot(x y)$, for all $\xi \in L^{0}(\mathcal{F}, K)$ and all $x, y \in S$;

(2) the $L^{0}$-norm $\|\cdot\|$ is submultiplicative, that is, $\|x y\| \leq\|x\|\|\mid\| y \|$, for all $x, y \in S$.

Furthermore, the RN algebra is said to be unital if it has the identity element $e$ and II $e \|=1$. As usual, the $\mathrm{RN}$ algebra $(S,\|\cdot\|)$ is said to be complete if the RN module $(S$, $\|\cdot\|)$ is complete.

Example 2.1. [5] Let $(X,\|\cdot\|)$ be a normed algebra over $C$ and $L^{0}(\mathcal{F}, X)$ be the RN module of equivalence classes of $X$-valued $\mathscr{F}$-random variables on $(\Omega, \mathcal{F}, P)$. Define a multiplication $: L^{0}(\mathcal{F}, X) \times L^{0}(\mathcal{F}, X) \rightarrow L^{0}(\mathcal{F}, X)$ by $x \cdot y=$ the equivalence class determined by the $\mathscr{F}$-random variable $x^{0} y^{0}$, which is defined by $\left(x^{0} y^{0}\right)(\omega)=\left(x^{0}(\omega)\right) \cdot\left(y^{0}(\omega)\right)$, $\forall \omega \in \Omega$, where $x^{0}$ and $y^{0}$ are arbitrarily chosen representatives of $x$ and $y$ in $L^{0}(\mathcal{F}, X)$, respectively. Then $\left(L^{0}(\mathcal{F}, X),\|\cdot\|\right)$ is an $\mathrm{RN}$ algebra, in particular $L^{0}(\mathcal{F}, C)$ is a unital RN algebra with identity 1 .

Example 2.2. [5] It is easy to see that $L_{\mathcal{F}}^{\infty}(\varepsilon, C)$ is a unital RN algebra with identity 1 (see $[8,9]$ for the construction of $L_{\mathcal{F}}^{\infty}(\varepsilon, C)$. 
Definition 2.4. [5] Let $(S,\|\cdot\|)$ be an RN algebra with identity $e$ over $C$ with base $(\Omega, \mathcal{F}, P)$, and $A$ be any given element in $\mathscr{F}$ such that $P(A)>0$. An element $x \in S$ is invertible on $A$ if there exists $y \in S$ such that $\tilde{I}_{A} \cdot x y=\tilde{I}_{A} \cdot y x=\tilde{I}_{A} \cdot e$. Clearly, $\tilde{I}_{A} \cdot y$ is unique and called the inverse on $A$ of $x$, denoted by $x_{A}^{-1}$. Let $G(S, A)$ denote the set of elements of $S$ which are invertible on $A$. Then $\tilde{I}_{A} \cdot G(S, A)$ is also a group, and $(x y)_{A}^{-1}=y_{A}^{-1} x_{A}^{-1}$ for any $x$ and $y$ in $\tilde{I}_{A} \cdot G(S, A)$. For any $x \in S$, the sets

$$
\begin{aligned}
\sigma(x, S, A) & =\left\{\xi \in L^{0}(\mathcal{F}, C): \tilde{I}_{A} \cdot(\xi \cdot e-x) \notin \tilde{I}_{A} \cdot G(S, A)\right\} \\
\sigma(x, S) & =\bigcap_{A \in \mathcal{F}} \sigma(x, S, A)
\end{aligned}
$$

are called the random spectrum on $A$ of $x$ in $S$ and the random spectrum of $x$ in $S$, respectively, and further their complements $\rho(x, S, A)=L^{0}(\mathcal{F}, C) \backslash \sigma(x, S, A)$ and $\rho(x, S)=L^{0}(\mathcal{F}, C) \backslash \sigma(x, S)$ are called the random resolvent set on $A$ of $x$ and the random resolvent set of $x$, respectively.

Definition 2.5. [5] Let $(S,\|\cdot\|)$ be an RN algebra with identity $e$ over $C$ with base $(\Omega, \mathcal{F}, P)$. For any $x \in S, r(x)=\mathrm{V}\{|\xi|: \xi \in \sigma(x, S)\}$ is called the random spectral radius of $x$.

Besides, $\wedge\left\{\left\|x^{n}\right\|^{\frac{1}{n}} \mid n \in N\right\}$ is denoted by $r_{p}(x)$, for any $x$ in an RN algebra over $K$ with base $(\Omega, \mathcal{F}, P)$.

Lemma 2.1. [5] Let $(S,\|\cdot\|)$ be a unital complete RN algebra with identity $e$ over $C$ with base $(\Omega, \mathcal{F}, P)$. Then for any $x \in S, \sigma(x, S)$ is nonempty and $r(x)=r_{p}(x)$.

\section{Main results and proofs}

Definition 3.1. Let $S$ be a random normed algebra, $A \in \mathcal{F}$ and $f$ be an $L^{0}$-linear function on $S$, i.e., a mapping from $S$ to $L^{0}(\mathcal{F}, C)$ such that $f(\xi \cdot x+\eta \cdot y)=\xi f(x)+\eta f(y)$ for all $\xi, \eta \in L^{0}(\mathcal{F}, C)$ and $x, y \in S$. Then $f$ is called multiplicative if $f(x y)=f(x) f(y)$ for all $x, y \in S$ and is called nonzero if there exists $x \in S$ such that $[f(x) \neq 0]=\tilde{\Omega}$.

Lemma 3.1. Let $S$ be a random normed algebra with identity $e$, and let $f$ be an $L^{0}$ function on $S$ satisfying $f(e)=1$ and $f\left(x^{2}\right)=f(x)^{2}$ for all $x \in S$. Then $f$ is multiplicative.

Proof. By assumption we obtain

$$
\begin{aligned}
f\left(x^{2}\right)+f(x y+y x)+f\left(y^{2}\right) & =f\left(x^{2}+x y+y x+y^{2}\right) \\
& =f\left((x+y)^{2}\right) \\
& =f(x+y)^{2} \\
& =f(x)^{2}+2 f(x) f(y)+f(y)^{2},
\end{aligned}
$$

and hence

$$
f(x y+y x)=2 f(x) f(y)
$$

for all $x, y \in S$. So it remains to verify that $f(x y)=f(y x)$. For $a, b \in S$, the identity

$$
(a b-b a)^{2}+(a b+b a)^{2}=2[a(b a b)+(b a b) a]
$$


implies

$$
\begin{aligned}
f(a b-b a)^{2}+4 f(a)^{2} f(b)^{2} & =f\left((a b-b a)^{2}\right)+f(a b+b a)^{2} \\
& =f\left((a b-b a)^{2}+(a b+b a)^{2}\right) \\
& =f\left((a b-b a)^{2}+(a b+b a)^{2}\right) \\
& =2 f(a(b a b)+(b a b) a) \\
& =4 f(a) f(b a b) .
\end{aligned}
$$

Taking $a=x-f(x) \cdot e$, so that $f(a)=0$, and $b=y$ we get $f(a y)=f(y a)$ and hence $f(x y)$ $=f(y x)$. This completes the proof of Lemma 3.1.

The following theorem is a new version of the Gleason-Kahane-Żelazko theorem.

Theorem 3.1 Let $S$ be an unital complete random normed algebra with identity $e$, and let $f$ be an $L^{0}$-linear function on $S$. Then the following conditions are equivalent.

(1) $f$ is nonzero and multiplicative.

(2) $f(e)=1$ and $f(x) \neq 0$ on $\tilde{A}$ for any $A \in \mathcal{F}$ with $P(A)>0$ and $x \in G(S, A)$.

(3) $f(x) \in \sigma(x, S)$ for every $x \in S$.

Proof If $f$ is multiplicative, then $f(e)=f\left(e^{2}\right)=f(e) f(e)$. Since $f$ is nonzero, we have $f(e)$ $=1$ and hence $\tilde{I}_{A}=\tilde{I}_{A} f(e)=f\left(x x_{A}^{-1}\right)=f(x) f\left(x_{A}^{-1}\right)$ for any $A \in \mathcal{F}$ with $P(A)>0$ and $x$ $\in G(S, A)$. Thus $(1) \Rightarrow(2) .(2) \Rightarrow(3)$ is clear since if $\xi \in \rho(x, S)$, then there exists $A \in \mathcal{F}$ with $P(A)>0$ such that $\tilde{I}_{A}(\xi-f(x))=f\left[\tilde{I}_{A} \cdot(\xi \cdot e-x)\right] \neq 0$ on $\tilde{A}$ and hence $f(x) \in \sigma$ $(x, S)$. Assume (3), then $f(e)=1$ since $f(e) \in \sigma(e, S)$. Now, let $n \geq 2$ and consider the random polynomial

$$
p(\lambda)=f\left((\lambda \cdot e-x)^{n}\right)
$$

of degree $n$. Therefore we can find $\lambda_{i} \in L^{0}(\mathcal{F}, C)(i=1,2 \ldots n)$ such that

$$
0=p\left(\lambda_{i}\right)=f\left(\left(\lambda_{i} \cdot e-x\right)^{n}\right) \in \sigma\left(\left(\lambda_{i} \cdot e-x\right)^{n}, S\right)
$$

for each $\lambda_{i}$. This implies that $\lambda_{i} \in \sigma(x, S)$ and hence $\left|\lambda_{i}\right|<r_{p}(x)$ by Lemma 2.1. Note that

$$
\prod_{i=1}^{n}\left(\lambda-\lambda_{i}\right)=p(\lambda)=\lambda^{n}-n f(x) \lambda^{n-1}+C_{n}^{2} f\left(x^{2}\right) \lambda^{n-2}+\cdots+(-1)^{n} f\left(x^{n}\right) .
$$

Comparing coefficients we can see that

$$
\sum_{i=1}^{n} \lambda_{i}=n f(x), \quad \sum_{1 \leq i<j \leq n} \lambda_{i} \lambda_{j}=C_{n}^{2} f\left(x^{2}\right) .
$$

On the other hand, by the second equation,

$$
\left(\sum_{i=1}^{n} \lambda_{i}\right)^{2}=\sum_{i=1}^{n} \lambda_{i}^{2}+2 \sum_{1 \leq i<j \leq n} \lambda_{i} \lambda_{j}=\sum_{i=1}^{n} \lambda_{i}^{2}+n(n-1) f\left(x^{2}\right) .
$$

Combining these equalities yields

$$
n^{2}\left|f(x)^{2}-f\left(x^{2}\right)\right|=\left|-n f\left(x^{2}\right)+\sum_{i=1}^{n} \lambda_{i}^{2}\right| \leq n\left|f(x)^{2}\right|+n r_{p}(x)^{2} .
$$


Hence

$$
\left|f(x)^{2}-f\left(x^{2}\right)\right| \leq \frac{1}{n}\left[\left|f\left(x^{2}\right)\right|+r_{p}(x)^{2}\right]
$$

Letting $n \rightarrow \infty$, we then obtain $f\left(x^{2}\right)=f(x)^{2}$ for all $x \in S$. It follows from Lemma 3.1 that $f$ is multiplicative. Clearly, $f$ is nonzero. Thus $(3) \Rightarrow(1)$. This completes the proof of Theorem 3.1.

Remark 3.1. When the base space $(\Omega, \mathcal{F}, P)$ of the $\mathrm{RN}$ module is a trivial probability space, i.e., $\mathcal{F}=\{\Omega, \not 0\}$, the new version of the Gleason-Kahane-Żelazko theorem automatically degenerates to the classical case.

\section{Acknowledgements}

This work was supported by the NSF of China under Grant No. 10871016.

\section{Author details}

${ }^{1}$ LMIB and School of Mathematics and Systems Science, Beihang University, Beijing 100191, P. R. China ${ }^{2}$ College of Mathematics Physics and Information Engineering, Jiaxing University, Jiaxing 314001, P. R. China

\section{Competing interests}

The author declares that they have no competing interests.

Received: 21 December 2011 Accepted: 13 April 2012 Published: 13 April 2012

\section{References}

1. Gleason, AM: A characterization of maximal ideals. J Anal Math. 19, 171-172 (1967)

2. Kahane, JP, Żelazko, W: A characterization of maximal ideals in commutative Banach algebras. Studia Math. 29, 339-343 (1968)

3. Jarosz, K: Generalizations of the Gleason-Kahane-Żelazko theorem. Rocky Mount J Math. 21(3):915-921 (1991)

4. Tang, YH: The Gleason-Kahane-Żelazko theorem in a complete random normed algebra. Acta Anal Funct Appl. (2011)

5. Tang, YH, Guo, TX: Complete random normed algebras. to appear

6. Dunford, N, Schwartz, JT: Linear Operators 1. Interscience. New York (1957)

7. Guo, TX: Some basic theories of random normed linear spaces and random inner product spaces. Acta Anal Funct Appl. 1, 160-184 (1999)

8. Guo, TX: Recent progress in random metric theory and its applications to conditional risk measures. Sci China Ser A. 54 633-660 (2011)

9. Guo, TX: Relations between some basic results derived from two kinds of topologies for a random locally convex module. J Funct Anal. 258, 3024-3047 (2010)

\section{Submit your manuscript to a SpringerOpen ${ }^{\circ}$ journal and benefit from:}

- Convenient online submission

Rigorous peer review

- Immediate publication on acceptance

- Open access: articles freely available online

- High visibility within the field

- Retaining the copyright to your article

Submit your next manuscript at $>$ springeropen.com 